Notfall Rettungsmed 2023 26:189-198 https://doi.org/10.1007/s10049-021-00959-w Angenommen: 14. Oktober 2021 Online publiziert: 2. Dezember 2021 ๑ ( Der/die Autor(en) 2021

\section{Notfallmedizinische Aspekte bei Laufveranstaltungen}

Simon-Richard Finke ${ }^{1}$ Christoph Jänig ${ }^{2} \cdot$ Andreas Deschler $^{2,3} \cdot$ Jan Hanske ${ }^{2}$. Holger Herff ${ }^{1}$ J Jochen Hinkelbein ${ }^{1}$ - Bernd W. Böttiger ${ }^{1}$ · W. Schmidbauer ${ }^{2}$. Daniel C. Schroeder ${ }^{1,2}$

${ }^{1}$ Klinik für Anästhesiologie und Operative Intensivmedizin, Universitätsklinikum Köln, Köln, Deutschland ${ }^{2}$ Klinik X - Anästhesie, Intensivmedizin und Notfallmedizin, Bundeswehrzentralkrankenhaus Koblenz, Koblenz, Deutschland

${ }^{3}$ Flugbereitschaft des Bundesministeriums der Verteidigung, Köln, Deutschland

Hintergrund: Die Anzahl an kurz- und langstreckigen Laufveranstaltungen in Deutschland nimmt zu. Laufen als Breitensport wird von einer Vielzahl an Personen unterschiedlicher Altersklassen, Risikogruppen und Professionalisierungsgrade betrieben, woraus ein breites Spektrum medizinischer Notfälle resultiert.

Ziel der Arbeit: Der vorliegende Beitrag erläutert die Inzidenz, Pathophysiologie und Therapie relevanter Notfallbilder bei Laufveranstaltungen. Ziel ist die Optimierung der Arbeitsabläufe des Rettungsdienstpersonals.

Material und Methoden: Es erfolgte eine Literaturrecherche in PubMed.

Ergebnisse: Anstrengungsassoziierte Muskelkrämpfe, gastrointestinale Symptome, Kollaps, Kompartmentsyndrom und Tendinopathien sind häufige Erscheinungsbilder und resultieren meist aus akuter oder chronischer Überanstrengung. Der Kreislaufstillstand bzw. plötzliche Herztod ist ein seltenes Ereignis bei Laufveranstaltungen. Bewusstseinsstörungen und generalisierte Krampfanfälle sind schwerwiegende Komplikationen. Disseminierte intravasale Koagulopathie, belastungsinduzierte Hyponatriämie, Hitzschlag, Rhabdomyolyse und thromboembolische Ereignisse sind mit hoher Morbidität und Mortalität verbunden. Substanzen zur Erhöhung der Schmerzschwelle und leistungssteigernde Substanzen sind unter Läufern stark verbreitet und gehen mit einer hohen Rate an Nebenwirkungen einher.

Diskussion: Unspezifische Symptome wie Erbrechen, Fieber, Kollaps, Muskelschmerzen, Übelkeit, und Schwäche sind die führenden Symptome bei Laufveranstaltungen. Eine sorgfältige Anamnese ist wegweisend für eine zielgerichtete klinische Therapie. Präklinisch steht eine Symptomkontrolle im Mittelpunkt. Das Flüssigkeitsmanagement stellt eine besondere Herausforderung dar.

\title{
Schlüsselwörter
}

Marathon · Laufen · Plötzlicher Herztod · Sportverletzungen · Wiederbelebung

\section{Hinführung zum Thema}

Das Angebot an kurz- und langstreckigen Laufveranstaltungen ist innerhalb der letzten 10 Jahre stark angestiegen. Einzelne Halbmarathonveranstaltungen verzeichnen bis zu 28.000 Teilnehmer. Im Jahr 2019 wurden in Deutschland 238 Marathonwettkämpfe durchgeführt, bei denen über 110.000 Teilnehmer das Ziel erreichten [3], was schätzungsweise $92 \%$ der Starter entspricht [4].
Laufen als Breitensport wird von einer Vielzahl an Personen unterschiedlicher Altersklassen, Risikogruppen und Professionalisierungsgrade betrieben. Aus dieser inhomogenen Population resultiert ein breites Spektrum medizinischer Notfälle, mit denen der Rettungsdienst auf Laufveranstaltungen konfrontiert wird. Zudem beeinflussen Wetterbedingungen, Unterhaltungs- und Rahmenprogramme sowie hohe Zuschaueraufkommen die Einsatzbilder. 
Tab. 1 Inzidenz von laufassoziierten muskuloskeletalen Verletzungen nach einer systematischen Übersichtsarbeit von Lopes et al. [14]

\begin{tabular}{|l|l|l|l|}
\hline \multicolumn{2}{|l|}{ Weichteilsystem } & Knöchernes System & $\begin{array}{l}\text { Inzidenz } \\
\mathbf{( \% )}\end{array}$ \\
\hline Verletzung & $\begin{array}{l}\text { Inzidenz } \\
(\%)\end{array}$ & Verletzung & 13,6-20,0 \\
\hline Patellasehnentendinopathie & $5,5-22,7$ & Mediales tibiales Stresssyndrom & $\mathbf{9 , 1}$ \\
\hline Achillessehnentendinopathie & $9,1-10,9$ & Verstauchung des Sprunggelenks & $10,9-15,0$ \\
\hline $\begin{array}{l}\text { Zerrung der Oberschenkelmusku- } \\
\text { latur }\end{array}$ & 10,9 & Stressfraktur der Tibia & 5 \\
\hline Muskelfaserriss & 10 & Verstauchung des Kniegelenks & $4,5-5,0$ \\
\hline Plantarfasziitis & $4,5-7,3$ & Weitere Frakturen & 5 \\
\hline
\end{tabular}

Der vorliegende Beitrag erläutert pathophysiologische Hintergründe und Therapieansätze präklinisch relevanter Notfallbilder bei Laufveranstaltungen. Ziel der Arbeit ist die Optimierung der Arbeitsabläufe von nichtärztlichem und ärztlichem Rettungsdienstpersonal und eine Vertiefung der Expertise der zu erwartenden Erkrankungen und Verletzungsmuster.

\section{Verletzungs- und Erkrankungs- bilder}

\section{Weichteilsystem}

\section{Anstrengungsassoziierte Muskelkrämpfe}

Als anstrengungsassoziierter Muskelkrampf (AAMK) wird eine schmerzhafte, spastische, unwillkürliche Skelettmuskelkontraktion bezeichnet, die während bzw. unmittelbar nach Belastung auftritt $[5,6]$. Als Ursache werden muskuläre Überlastung [5], Elektrolytverschiebungen [6] und eine geringe Trinkmenge diskutiert $[6,7]$. Eine Häufung des AAMK zeigt sich bei älteren und untrainierten Sportlern sowie auf dem Boden einer

\section{Abkürzungen}

\begin{tabular}{ll} 
AAMK & $\begin{array}{l}\text { Anstrengungsassoziierter Muskel- } \\
\text { krampf } \\
\text { Anabole androgene Steroide }\end{array}$ \\
COX & $\begin{array}{l}\text { Cyclooxygenase } \\
\text { Disseminierte intravasale Koagulo- } \\
\text { pathie (Gerinnung) }\end{array}$ \\
& $\begin{array}{l}\text { International Liaison Committee on } \\
\text { ILCOR }\end{array}$ \\
RHK & Koronscitation \\
NSAR & Nichtsteroidales Antirheumatikum \\
NSTEMI & Nicht-ST-Strecken-Elevations- \\
& Myokardinfarkt \\
STEMI & ST-Strecken-Elevations-Myokardin- \\
& farkt \\
\hline
\end{tabular}

Gelenkfehlstellung [5, 6]. In Muskelgruppen mit starker Kontraktionsfähigkeit und hoher Beanspruchung treten ebenfalls vermehrt AAMK auf [6]. Charakteristisch sind anhaltende, schmerzhafte Muskelkontraktion und Bewegungsunfähigkeit der beanspruchten Muskelgruppe während oder unmittelbar nach Belastung [5, 6]. Therapeutisch werden eine vorsichtige Dehnung des Muskels und die orale Rehydrierung empfohlen $[5,6]$.

\section{Tendinopathien}

Aus Überbeanspruchung der Sehnen resultiert eine progressive Degeneration von Kollagenfasern [8, 9]. Aufgrund fehlender Reparaturmaßnahmen kommt es zu Schmerz bzw. Funktionsverlust (Tendinopathie). Dieser muss nicht zwangsläufig mit einem strukturellen Schaden - z. B. einem Riss - einhergehen [9]. Eine Achillessehnentendinopathie zeigt sich bei Kurzstreckenläufern gehäuft [10]. Die Plantarfasziitis geht mit einer Degeneration der Plantarfaszie einher und ist der häufigste Grund für Schmerzen der Ferse ([11]; - Tab. 1). Klinisch zeigt sich ein häufig vorbekannter Ruhe- oder Belastungsschmerz [9-11]. Die präklinische Therapie besteht in Schonung der betroffenen Extremität und Analgesie [12, 13].

Merke Verletzungen des Weichteilapparats gehen häufig mit unspezifischen Symptomen einher [10]. Die präklinische Diagnose ist schwierig. Die Beendigung des Wettkampfs kann folgenreiche Schäden verhindern.

\section{Rhabdomyolyse}

Eine Rhabdomyolyse kann durch Traumata, Medikamente und Noxen sowie eine ausgeprägte thermische Belastung
(Hitzschlag) ausgelöst werden [15]. Der Rhabdomyolyse liegt eine Schädigung der Zellmembran der Skelettmuskelzelle (Sarkolemm) oder ein Zusammenbruch der Energieversorgung mit Dysfunktion der $\mathrm{Na}^{+} / \mathrm{K}^{+}$- und $\mathrm{Ca}^{++}$-ATPase zugrunde. Es resultiert eine erhöhte Permeabilität für $\mathrm{Na}^{+}$nach intrazellulär mit konsekutivem Anstieg der intrazellulären $\mathrm{Ca}^{++}$-Konzentration. Nach Aktivierung $\mathrm{Ca}^{++}$-abhängiger Proteasen und Phospholipasen kommt es zur Schädigung von Zellstrukturen mit Freisetzung von Proteinen (Myoglobin, Kreatininkinase, Laktatdehydrogenase) und Metaboliten ( $\mathrm{K}^{+}$, Phosphat; [15]). Erhöhte Serummyoglobinspiegel führen zur mechanischen Verlegung der Nierentubuli und begünstigen ein akutes Nierenversagen $[15,16]$. Zudem können freigesetzte Proteine und Elektrolyte zu Herzrhythmusstörungen, Kreislaufstillstand, einer disseminierten intravasalen Gerinnung oder einer Ödematisierung der Muskulatur mit Kompartmentsyndrom führen [15]. Unspezifische Anfangssymptome wie Schmerzen, Schwäche und Bewegungseinschränkungen weisen auf eine Schädigung der Skelettmuskulatur hin, deren Ausmaß nicht unterschätzt werden darf. Präklinisch sollte eine symptomatische Therapie (Analgesie, Kühlung) erwogen werden [15]. Bei unklarer Diagnose sollte eine großzügige Einweisung ins Krankenhaus erfolgen.

Merke Hinweise auf eine größere Schädigung der Skelettmuskulatur benötigen eine serologische Abklärung zum Ausschluss von Nierenschäden und Elektrolytstörungen im Krankenhaus.

\section{Knöchernes System}

\section{Frakturen}

Chronische Überbeanspruchung des Knochens führt zunächst zu einer Verschiebung des Gleichgewichts im Knochenstoffwechsel mit vermehrtem Knochenabbau. Bei fortgesetzter Belastung unterhalb der Frakturschwelle entstehen Mikrofrakturen, die bei ausbleibenden Reparaturmechanismen in einer (Stress-)Fraktur münden ([17]; - Tab. 1). Betroffen sind vorwiegend Tibia, Fibula, Mittelfußknochen sowie der Beckenkamm [18]. Klinisch zeigt sich ein vorbestehender, zunehmender Be- 
Tab. 2 Verletzungen der Gelenke. (Modifiziert nach [1 2])

\begin{tabular}{|l|l|l|l|}
\hline & Prellung (Kontusion) & Zerrung/Dehnung (Distorsion) & Verrenkung (Luxation) \\
\hline $\begin{array}{l}\text { Pathome- } \\
\text { chanismus }\end{array}$ & $\begin{array}{l}\text { Direkte stumpfe Gewalteinwir- } \\
\text { kung ohne Verschiebung der } \\
\text { Gelenkflächen gegeneinander }\end{array}$ & $\begin{array}{l}\text { Verschiebung der gelenkbildenden Teile mit Überschrei- } \\
\text { tung des physiologischen Bewegungsumfangs und an- } \\
\text { schließender Rückkehr in die Ausgangsposition }\end{array}$ & $\begin{array}{l}\text { Dauerhafte Verschiebung der gelenk- } \\
\text { bildenden Elemente (vollständig/ } \\
\text { unvollständig) }\end{array}$ \\
\hline $\begin{array}{l}\text { Geschädig- } \\
\text { te Struktur }\end{array}$ & Schäden am Gelenkknorpel & Überdehnung der Gelenkkapsel & Schädigungen des Kapselbandapparats \\
\cline { 2 - 4 } & Einblutung in den Gelenkraum & Schäden/Einblutung der Gelenkkapsel möglich & Knorpel-, Knochenverletzungen \\
\hline
\end{tabular}

Tab. 3 Klinik und Therapie relevanter notfallmedizinischer Krankheitsbilder bei Laufveranstaltungen

\begin{tabular}{|c|c|c|}
\hline $\begin{array}{l}\text { Akutes Koronarsyn- } \\
\text { drom (ACS) }\end{array}$ & $\begin{array}{l}\text { Atemnot, akuter Brustschmerz, STEMI, NSTEMI, Arrhythmien, Leis- } \\
\text { tungseinschränkung, Übelkeit, Erbrechen, Schwitzen, Synkope, Lun- } \\
\text { genödem, Zyanose [19] }\end{array}$ & Leitlinienbasierte Therapie [19] \\
\hline $\begin{array}{l}\text { Belastungsinduzierte } \\
\text { Hyponatriämie }\end{array}$ & $\begin{array}{l}\text { Lungenödem, neurologische Symptomatik (siehe akutes Hirnödem), } \\
\text { präklinisch Ausschlussdiagnose }[20,21]\end{array}$ & $\begin{array}{l}\text { Zurückhaltende Substitution mit Vollelektrolytlö- } \\
\text { sung [19] }\end{array}$ \\
\hline Frakturen & Gelenkfehlstellung, Krepitation, Ruhe- und Belastungsschmerz [17] & Schienung, Analgesie, ggf. Reposition [17] \\
\hline Akutes Hirnödem & $\begin{array}{l}\text { Milde Symptomatik: Kopfschmerzen, Schwindel, Übelkeit, Erbrechen, } \\
\text { retrograde Amnesie, Verwirrtheit; schwere Symptomatik: Bewusst- } \\
\text { seins- und Atemstörungen, Cushing-Reflex, Lähmungen, neurogenes } \\
\text { Lungenödem, Pupillendifferenz, Beuge- und Strecksynergismen, } \\
\text { Krampfanfall }[20,22]\end{array}$ & Symptomatisch, Sicherung der Vitalfunktionen \\
\hline Hypoglykämie & $\begin{array}{l}\text { Übelkeit, Erbrechen, Vigilanzminderung, Verwirrtheit, Unruhe, Schwä- } \\
\text { che, Krampfanfall [23] }\end{array}$ & $\begin{array}{l}\text { Sicherung der Vitalfunktionen, intravenöse Zufuhr } \\
\text { von Glukose [23] }\end{array}$ \\
\hline Kreislaufstillstand & Atemstillstand, Bewusstlosigkeit, fehlende Lebenszeichen $[24,25]$ & Herz-Lungen-Wiederbelebung nach Leitlinien [25] \\
\hline $\begin{array}{l}\text { Lungenarterien- } \\
\text { embolie }\end{array}$ & $\begin{array}{l}\text { Plötzliche Luftnot, Tachykardie, Brustschmerz, Husten, ggf. blutiger } \\
\text { Auswurf, hämodynamische Instabilität, rechtsventrikuläre Belastung, } \\
\text { Trikuspidalinsuffizienz [26] }\end{array}$ & $\begin{array}{l}\text { Sofortige therapeutische Antikoagulation, Kate- } \\
\text { cholamine, moderate Flüssigkeitsgabe, } \mathrm{O}_{2} \text {-Appli- } \\
\text { kation, umgehende Einweisung mit Reperfusions- } \\
\text { möglichkeit, instabile Patienten: Lyse erwägen [26] }\end{array}$ \\
\hline $\begin{array}{l}\text { Orthostatische Dys- } \\
\text { regulation }\end{array}$ & $\begin{array}{l}\text { Hypotension mit (hyperadrenerge Form) und ohne Tachykardie (hy- } \\
\text { poadrenerge Form) bzw. mit Bradykardie (neurokardiogene Form), } \\
\text { Bewusstlosigkeit }[22,27]\end{array}$ & $\begin{array}{l}\text { Konservative Maßnahmen: Sympathomimetika, } \\
\text { orale Flüssigkeitszufuhr, Trendelenburg-Lagerung, } \\
\text { „begleitetes Gehen“ zur Aktivierung der Muskel- } \\
\text { pumpe (cave: Hypertension im Liegen; [28]) }\end{array}$ \\
\hline $\begin{array}{l}\text { Schienbeinkanten- } \\
\text { syndrom }\end{array}$ & $\begin{array}{l}\text { Belastungsschmerz im distalen Bereich der posteromedialen Tibia- } \\
\text { kante [29] }\end{array}$ & Schonung, Analgesie [29] \\
\hline $\begin{array}{l}\text { Schwerwiegender } \\
\text { Kollaps }\end{array}$ & $\begin{array}{l}\text { Tachykardie, Hyperthermie, metabolische Azidose, Hypernatriämie } \\
\text { [30] }\end{array}$ & Symptomatisch, Stabilisierung des Kreislaufs [30] \\
\hline $\begin{array}{l}\text { Tiefe Beinvenen- } \\
\text { thrombose }\end{array}$ & $\begin{array}{l}\text { Schmerzen, Krämpfe, Ödematisierung, Überwärmung, Zyanose, livide } \\
\text { Verfärbung, Ödem [26] }\end{array}$ & $\begin{array}{l}\text { Symptomatische Therapie, klinischer Ausschluss } \\
\text { Lungenarterienembolie [26] }\end{array}$ \\
\hline $\begin{array}{l}\text { Traumatische Blu- } \\
\text { tung }\end{array}$ & $\begin{array}{l}\text { Tachykardie, Hypotension, Kaltschweißigkeit, Rekapillarisierungszeit } \\
>2 \mathrm{~s} \text { [31] }\end{array}$ & $\begin{array}{l}\text { Blutstillung (Kompression, Tourniquet, Becken- } \\
\text { schlinge), Tranexamsäure erwägen [31] }\end{array}$ \\
\hline $\begin{array}{l}\text { Herzrhythmus- } \\
\text { störungen (HRST) }\end{array}$ & $\begin{array}{l}\text { Tachykarde HRST: > 100/min; Bradykarde HRST: <60/min; Palpitatio- } \\
\text { nen, Synkope, Übelkeit, Erbrechen, ACS, Herzinsuffizienz [25] }\end{array}$ & $\begin{array}{l}\text { Symptomatisch, Stabilisierung des Kreislaufs, siehe } \\
\text { ILCOR-Leitlinien [25] }\end{array}$ \\
\hline Prellung (Kontusion) & $\begin{array}{l}\text { Druckschmerz, Weichteilschwellung, Bewegungseinschränkung [12, } \\
\text { 13] }\end{array}$ & $\begin{array}{l}\text { Analgesie, abschwellende Maßnahmen, Ruhigstel- } \\
\text { lung }[12,13]\end{array}$ \\
\hline $\begin{array}{l}\text { Zerrung/Dehnung } \\
\text { (Distorsion) }\end{array}$ & $\begin{array}{l}\text { Schmerz über der betr. Bandstruktur, Weichteilschwellung, Bewe- } \\
\text { gungseinschränkung, Dehnungsschmerz }[12,13]\end{array}$ & $\begin{array}{l}\text { Analgesie, abschwellende Maßnahmen, Ruhigstel- } \\
\text { lung }[12,13]\end{array}$ \\
\hline $\begin{array}{l}\text { Verrenkung (Luxati- } \\
\text { on) }\end{array}$ & $\begin{array}{l}\text { Gelenkfehlstellung, leere Gelenkpfanne, Schmerz, Schwellung, Bewe- } \\
\text { gungseinschränkung }[12,13]\end{array}$ & $\begin{array}{l}\text { Reposition bei Verlust der Durchblutung, Sensibi- } \\
\text { lität erwägen, Klinikeinweisung, Analgesie, Ruhig- } \\
\text { stellung }[12,13]\end{array}$ \\
\hline
\end{tabular}

STEMI ST-Strecken-Elevations-Myokardinfarkt, NSTEMI Nicht-ST-Strecken-Elevations-Myokardinfarkt, ILCOR International Liaison Committee on Resuscitation

lastungsschmerz, der durch Palpation lokalisierbar ist und von unspezifischen Symptomen wie Rötung und Schwellung begleitet wird [18]. Schonung, Schienung und Analgesie stellen präklinische Therapieansätze dar [18]. Die Fortsetzung des Wettkampfs kann nicht empfohlen werden. Darüber hinaus kann es bei Laufveranstaltungen zu Frakturen und Gelenkverletzungen (- Tab. 2 und 3) infolge akuter Kraft- bzw. Gewalteinwirkung kommen. Erschöpfung, Unachtsamkeit oder vorbestehende gesundheitliche Einschränkungen können einen Sturz bewir- ken und müssen anamnestisch abgeklärt werden.

\section{Kompartmentsyndrom}

Traumata und wiederkehrende hohe körperliche Anstrengung führen zur Volumenzunahme in den Muskellogen. 
Die Volumenzunahme verursacht einen akuten oder chronischen interstitiellen Druckanstieg mit Beeinträchtigung der Gewebsperfusion. Entstandenes ischämisches Muskelgewebe führt zur Ausbildung eines Ödems, sodass Druckanstieg und Minderperfusion verstärkt werden. Im Rahmen von Laufveranstaltungen ist vor allem die vordere Schienbeinmuskulatur betroffen [29]. Die Symptome sind zunächst unspezifisch (Krämpfe, Muskelschwäche). Später fällt eine klinische Härtung des Muskels bei Bewegung mit begleitendem Ruheschmerz, Hypästhesie und Minderdurchblutung auf [29, 32]. Neben Schonung und Analgesie müssen Durchblutung, Motorik, Sensibilität (DMS) überprüft werden [29, 32]. Das fortgeschrittene Kompartmentsyndrom erfordert eine zeitnahe chirurgische Intervention in der Klinik.

Merke Die präklinische Diagnose des Kompartmentsyndroms ist entscheidend für die Prognose des Patienten [29].

\section{Herz-Kreislauf-System}

\section{Plötzlicher Herztod/ \\ Kreislaufstillstand}

Die Inzidenz des Kreislaufstillstands bei Laufveranstaltungen liegt zwischen 1:15.000 und 1:150.000 [33-35]. Die Datenlage zu Überlebensraten ist schwach. Kim et al. berichten, dass $71 \%$ der Teilnehmer eines Halbmarathons bzw. Marathons nach Kreislaufstillstand versterben, was einer Inzidenz von einem Todesfall pro 259.000 Teilnehmer entspricht [34]. Webner et al. zeigten in einer retrospektiven Analyse eine Inzidenz von einem Todesfall pro 171.005 Teilnehmer [36]. Der Kreislaufstillstand betrifft vor allem Männer (93\%) mit einem Durchschnittsalter von 45 bis 50 Jahren, ist in 65-70\% mit einer koronaren Herzkrankheit (KHK) vergesellschaftet und tritt gehäuft auf den letzten $5 \mathrm{~km}$ eines Marathons auf [34, 36]. Eine vorbestehende hypertrophe Kardiomyopathie geht mit einer hohen Mortalität bei Laufveranstaltungen einher [34] und ist die Hauptursache für Kreislaufstillstände bei jüngeren Sportlern [37]. Hyponatriämie und Hitzschlag sind seltene Gründe für einen Kreislaufstillstand [34]. Ein früher Beginn der Wiederbelebungsmaßnahmen und initiales Kammerflimmern sind Prädiktoren für die Wiederherstellung eines Kreislaufs [34].

Merke Die Sicherstellung von Sanitätsund Rettungsdienstpersonal sowie die Möglichkeit der (Früh-)Defibrillation auf den letzten $10 \mathrm{~km}$ eines Marathons können die Überlebensrate steigern [34].

Pathophysiologisch existieren verschiedene Erklärungsmodelle für die Entstehung eines Kreislaufstillstands bei Laufveranstaltungen. Durch Rhabdomyolyse und Flüssigkeitsverlust kommt es zu Elektrolytverschiebungen, woraus hämodynamisch relevante kardiale Arrhythmien resultieren (• Tab. 3; [15, 38]). Zudem begünstigt infolge körperlicher Aktivität freigesetztes Interleukin-6 das Syndrom der inadäquaten Ausschüttung von antidiuretischem Hormon, was wiederum die Natriumausscheidung fördert und eine Hyponatriämie begünstigt $[20,39]$.

Darüber hinaus trägt eine belastungsinduzierte Zytokinausschüttung zur Ruptur instabiler Plaques in den Herzkranzgefäßen bei, was durch steigende Troponinwerte bereits bei asymptomatischen Läufern abgebildet wird [40]. Eine relative Dehydratation sowie eine belastungsbedingte prokoagulatorische Blutgerinnung erhöhen das Risiko für kardiale Ereignisse im Vergleich zur Normalbevölkerung nach einem Langstreckenlauf temporär [39]. Die novellierten Wiederbelebungsleitlinien empfehlen, dass der Kollaps eines Sportlers eine „unmittelbare Reaktion ... des medizinischen Teams" auslösen sollte. In diesem Rahmen müssen im Vorfeld Zugangswege zum Patienten für den Rettungsdienst geschaffen werden und bekannt sein. Die Möglichkeit zur (Laien-)Defibrillation muss jederzeit bestehen. Die Laienausbildung aller Sportler in Wiederbelebungsmaßnahmen soll gesteigert werden. Bei erhöhtem Zuschaueraufkommen kann ein Transport in eine geeignete Umgebung nach drei aufeinanderfolgenden Defibrillationsversuchen unter Fortführung effektiver Thoraxkompressionen erwogen werden. Die Wiederbelebungsmaßnahmen sollen dem allgemeinen Wiederbelebungsalgorithmus folgen [41].

\section{Kollaps}

Der Kollaps in Folge einer klassischen orthostatischen Dysregulation ist mit 59\% der medizinischen Kontakte die häufigste Komplikation bei Langstreckenläufen. Ein Großteil der Patienten benötigt keine medizinische Betreuung. Wird diese benötigt, spricht man von einem „schwerwiegenden Kollaps" [30], der mit Tachykardie, Hyperthermie, metabolischer Azidose und Hypernatriämie als Zeichen der temporären Dehydratation einhergeht. Präklinisch stehen die ausführliche Anamnese und die Sicherung der Vitalfunktionen nach dem ABCDE-Schema im Mittelpunkt.

\section{Wasser-, Elektrolythaushalt}

\section{Belastungsinduzierte Hypo- natriämie}

Ein Serumnatriumspiegel von $<135 \mathrm{mmol} / \mathrm{I}$ wird als Hyponatriämie definiert und tritt bei $13 \%$ aller Langstreckenläufer auf [22]. Eine belastungsinduzierte Hyponatriämie verläuft meist asymptomatisch und ist spontan reversibel [20, 27]. Folgende Mechanismen tragen zur Entstehung bei:

- Vermehrter oraler Konsum von hypotoner Flüssigkeit (hypervolämische Hyponatriämie; [20, 30])

- Belastungsinduzierte Hyperthermie mit Abgabe von Wärmeenergie über Verdunstung (Evaporation), in deren Folge es zu Flüssigkeitsverlust mit Dehydratation und Hyponatriämie (hypovolämische Hyponatriämie) kommt $[20,30]$

Von einer schweren (belastungsinduzierten) Hyponatriämie spricht man ab einem Serumnatriumspiegel von $<125 \mathrm{mmol} / \mathrm{l}$ [42]. Aufgrund einer entstehenden intrazellulären Flüssigkeitszunahme mit Ödematisierung der Zellen besteht die Gefahr eines akuten Hirnödems mit Anstieg des intrakraniellen Drucks [20]. In diesem Rahmen kommt es zunächst zu unspezifischen Symptomen wie Übelkeit, Erbrechen, Ataxie, und Orientierungsstörungen. Quantitative Bewusstseinsstörungen, generalisierte Krampfanfälle und Lungenödem mit Atemnot sind Symptome der schweren Hyponatriämie [1, 42].

Die klinische Diagnose der belastungsinduzierten Hyponatriämie ist äußerst 


\section{Infobox 1}

Empfehlungen zum Umgang mit dehydrierten Patienten. (Mod. nach [1] und

[2])

- Symptomkontrolle/Sicherung der Vitalfunktionen

- Denke an eine Hyponatriämie als Differenzialdiagnose.

- Achte bei der Untersuchung (ABCDE-Schema) auf eine gewissenhafte Beurteilung des neurologischen Status.

- Messe den Blutzuckerspiegel.

- Erwäge Point-of-Care-Diagnostik.

- Vermeide unkontrollierte Flüssigkeitszufuhr (restriktives Flüssigkeitsmanagement).

- Verwende 0,9\%ige $\mathrm{NaCl}$-Lösung oder balancierte Vollelektrolytlösungen zur intravenösen Substitution.

- Überwache Patienten mit milden Symptomen mindestens $60 \mathrm{~min}$. Stelle eine großzügige Indikation zur Messung des Serumnatriumspiegels, was meist mit der Einweisung ins Krankenhaus verbunden ist.

- Substituiere 10-20 $\mathrm{ml} / \mathrm{kg}$ einer geeigneten Infusionslösung bei klinisch instabilen Patienten (z. B. anhaltende Hypotonie).

- Weise Patienten mit nicht eindeutig einzuordnender Symptomatik und schweren Verläufen großzügig ins Krankenhaus ein.

schwierig, da sie leicht mit einem Hitzschlag oder einer Dehydratation verwechselt werden kann $[2,43]$. Eine exakte präklinische Differenzierung des Schweregrads ist unmöglich. Nur durch Bestimmung des Serumnatriumspiegels kann die Diagnose der belastungsinduzierten Hyponatriämie gestellt werden [2]. Die gewissenhafte klinische Untersuchung nach dem ABCDESchema und die sorgfältige Erhebung des neurologischen Status haben daher einen hohen Stellenwert in der präklinischen Einschätzung von Patienten bei Laufveranstaltungen. Differenzialdiagnostisch sollte eine Hypoglykämie präklinisch ausgeschlossen werden [2].

Merke Bei der Betreuung einer großen Anzahl an Patienten sollte insbesondere auf das Auftreten von Bewusstseinsstörungen und generalisierten Krampfanfällen geachtet werden, die Ausdruck fortgeschrittener Elektrolytstörungen sind und auch mit Latenz nach Beendigung der Belastung auftreten können [2].

Die Symptomkontrolle steht im Mittelpunkt therapeutischer Ansätze bei Lauf- veranstaltungen (- Tab. 3; [2, 44]). Nach Beendigung der Belastung kann es zur weiteren Absorption von Flüssigkeit aus dem Gastrointestinaltrakt kommen, was in der Zunahme der Symptome münden kann [2]. Daher sollte bereits bei Verdacht auf eine milde Hypotonie eine lückenlose Überwachung und zeitnahe Einweisung ins Krankenhaus erfolgen. Patienten mit Verdacht auf eine schwere Hyponatriämie bedürfen einer schnellen intensivmedizinischen Therapie.

Das Flüssigkeitsmanagement stellt eine besondere Herausforderung unter präklinischen Bedingungen dar. Eine rasche Volumentherapie geht mit der Gefahr des weiteren Abfalls des Serumnatriumspiegels einher. Grundlage für eine adäquate Flüssigkeitssubstitution (Rehydratation) ist daher die Kenntnis des Serumelektrolytspiegels, was präklinisch nur erschwert möglich ist [2].

Bei Patienten mit milden Symptomen sollte die Flüssigkeitszufuhr oral erfolgen $[44,45]$. Die intravenöse Flüssigkeitszufuhr sollte kritisch kranken Patienten mit einer schweren Dehydratation (Verlust von > 7\% des Körpergewichts, Hypotonie) oder eingeschränkter oraler Aufnahme (Übelkeit, Erbrechen) vorbehalten bleiben und unter engmaschiger klinischer Überwachung durchgeführt werden [44, 45]. Zur intravenösen Substitution können 0,9\% NaCl-Lösung oder balancierte Vollelektrolytlösungen verwendet werden ([1]; • Infobox 1).

Merke Schnelle Marathonläufer nehmen während des Laufs geringere Mengen Flüssigkeit zu sich, während bei langsameren Läufern ein „Übertrinken“ beobachtet werden konnte. Schnelle Läufer sind eher durch Dehydratation, langsame Läufer eher durch eine Hyponatriämie gefährdet [45].

\section{Hitzschlag}

Dem Hitzschlag liegt eine Imbalance zwischen Wärmeproduktion und -verlust zugrunde, die durch Aufenthalt in warmer Umgebung oder starke körperliche Aktivität ausgelöst wurde. Der Hitzschlag ist charakterisiert durch eine Körperkerntemperatur von $>40^{\circ} \mathrm{C}$, Krampfanfälle, Bewusstseinsstörungen und multiples Organversagen [46]. Unabhängig von der Sportart stellt der Belastungshitzschlag bei jun- gen Sportlern mit einer Mortalität von 21 bis $63 \%$ die zweithäufigste Todesursache dar (- Abb. 1; [16, 47, 48]). Entkleidung und dermale Kühlung (Eis, Tücher) sind Eckpunkte der präklinischen Therapie. Sicherung der Vitalfunktionen und symptomatische Therapie haben eine hohe Bedeutung. Bei hoher Körpertemperatur und vital bedrohlichen klinischen Symptomen muss eine zügige Einweisung ins Krankenhaus erfolgen.

Merke Der Hitzschlag ist ein bedrohliches Krankheitsbild [46]. Bei Laufveranstaltungen muss bei allen Patienten die Körpertemperatur gemessen werden.

\section{Nierenfunktion}

Das Risiko, im Rahmen einer kurz- oder langstreckigen Laufveranstaltung eine Nierenfunktionseinschränkung zu entwickeln, ist beim gesamten Teilnehmerfeld erhöht [16]. Die Ursachen beinhalten

- eine katecholamininduzierte renale Vasokonstriktion mit resultierender Reduktion der glomerulären Filtrationsrate [16, 22],

- eine Einnahme von NSAR mit Einschränkung der renalen Durchblutung $[16,49]$,

- eine Reduktion des Plasmavolumens mit Aktivierung der Arginin-Vasopressin-Achse, die zu Flüssigkeitsretention und Oligurie führt [16, 22],

- das Einsetzen einer Rhabdomyolyse mit Freisetzung von Proteinen und Metaboliten [16, 22].

Die präklinische Diagnose der akuten Nierenfunktionsstörung ist aufgrund der heterogenen Klinik schwierig. Häufig zeigen sich symptomlose Verläufe. Weitere Manifestationen sind Kollaps, Elektrolytstörungen, Herzrhythmusstörungen, Oligurie, Polyurie, Anurie, metabolische Azidose, zentrale/periphere Ödeme und Muskelschwäche $[16,50]$. Eine symptomatische Therapie, die Stabilisierung und eine zeitnahe Einweisung des Patienten sind wegweisend.

\section{Gerinnungssystem}

Langstreckenläufer unterliegen einem erhöhten Risiko für thromboembolische Ereignisse. Traumata der Gefäßwand, systemische Entzündungsreaktionen und De- 


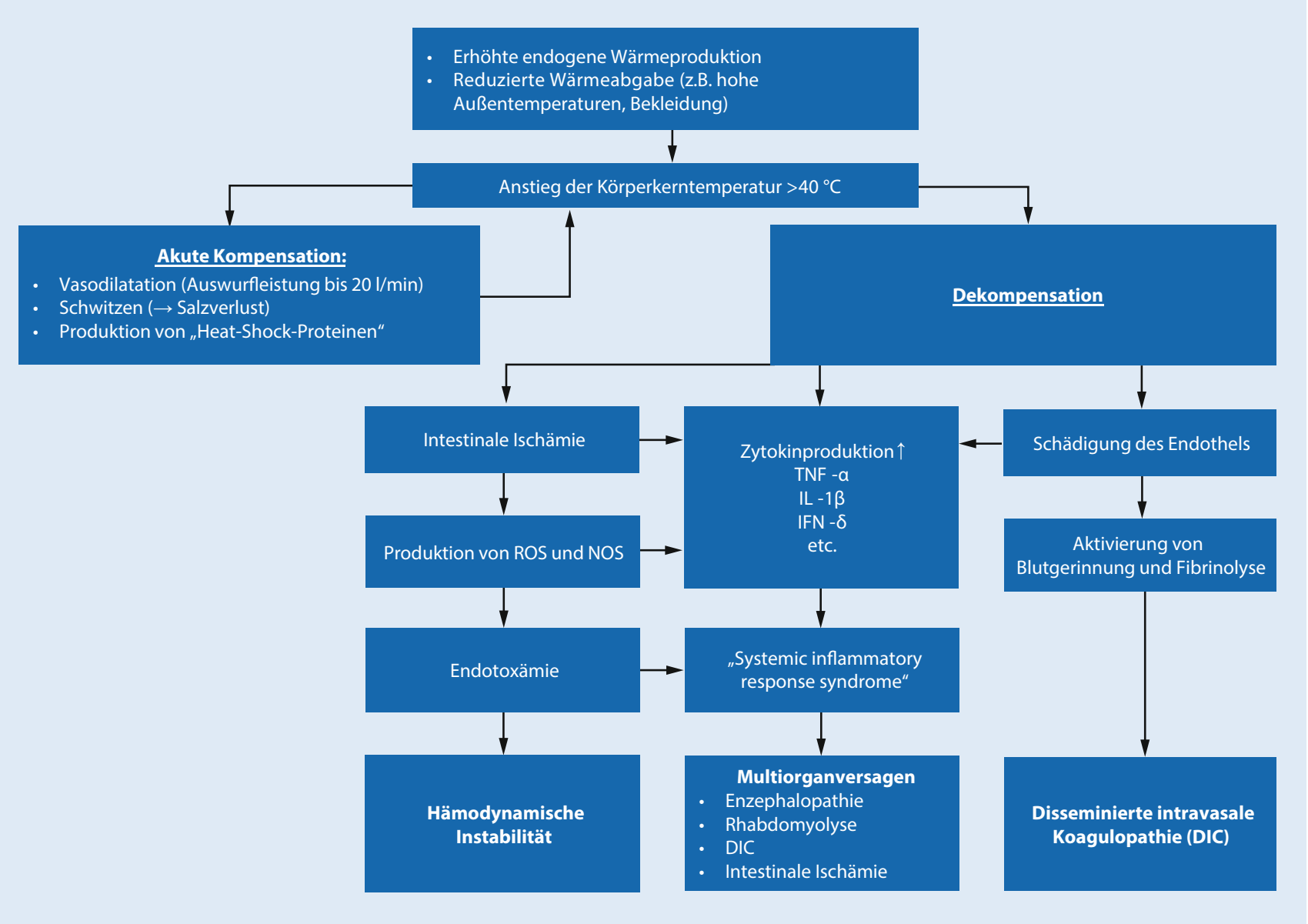

Abb. 1 \& Pathophysiologie des Hitzschlags. Mit Anstieg der Körperkerntemperatur $>40^{\circ} \mathrm{C}$ führen Dekompensationsmechanismen zum Multiorganversagen mit hoher Letalität. ROS "reactive oxygen species", NOS "nitric oxygen species", TNFTumornekrosefaktor, IL Interleukin, IFN Interferon. (Modifiziert nach Liu et al. [46])

hydratation verändern die Viskosität des Blutes. Belastungsinduzierte Bradykardie sowie Blutdruckanstiege und -abfälle verändern den Blutfluss [51]. Klinisch sind Verschlüsse der Lungenarterien [52] und der Herzkranzgefäße [53] sowie die Zentralvenenthrombose der Retina [54] nach einer Laufveranstaltung beschrieben. Im Rahmen des Hitzschlags wurde zudem von der Entwicklung einer disseminierten intravasalen Koagulopathie (DIC) berichtet [55].

\section{Gastrointestinaltrakt}

Das Auftreten von gastrointestinalen Symptomen bei Ausdauerläufern wird mit 30-90\% angegeben (• Tab. 3; [56]). Die Schwere der Symptomatik korreliert mit der Distanz bzw. dem Ausmaß der körperlichen Belastung [56]. Ursächlich ist eine belastungsabhängige Sympathikusaktivierung mit Vasokonstriktion der
Gefäße im Splanchnikusgebiet. Der intestinale Blutfluss wird konsekutiv um bis zu $80 \%$ reduziert, was eine Ischämie der Darmmukosa begünstigt [56]. Bei hoher Belastung wird die gastrointestinale Motilität gehemmt und die intestinale Flüssigkeitsabsorption reduziert [57], was die Aspirationsgefahr bei Bewusstlosigkeit erhöht. Die Aufnahme von protein-, kohlenhydrat- und fettreicher Spezialnahrung fördert das Auftreten gastrointestinaler Symptome [56]. Leicht symptomatische Patienten zeigen Diarrhö, Defäkationsdrang, Erbrechen, Flatulenz, gastroösophagealen Reflux, Übelkeit, Krämpfe und Koliken. Ein Übergang zu schweren Symptomen wie abdomineller Angina, blutigen Durchfällen, Dünndarminfarzierung, hämorrhagischer Gastritis, ischämischer Kolitis und rektaler (okkulter) Blutung ist möglich $[56,57]$. Die symptomatische The- rapie und eine vorsichtige Rehydrierung sind präklinisch indiziert [56].

\section{Läuferspezifisches Risikoprofil}

Sportliche Aktivität ist Bestandteil der Sekundärprophylaxe von Bluthochdruck, KHK und Diabetes mellitus. Amateurläufer zeigen häufig ein entsprechendes Risikoprofil, was bei der Patientenversorgung anamnestisch abgeklärt werden muss. Krankheitsbezogene Laufgruppen nehmen regelmäßig gemeinschaftlich an Laufveranstaltungen teil.

Ausdauersport kann bei Patienten mit Diabetes mellitus zu einer symptomatischen Hypoglykämie mit der Ausbildung einer Ketoazidose und neurologischer Symptomatik führen. Automatisierte Zuckersensoren und Insulinpumpen finden unter Sportlern breite Anwendung und können aufgrund von Fehlfunk- 
tionen oder fehlerhafter Bedienung zu rettungsdienstlichem Kontakt führen. Einige Laufveranstaltungen bieten parallel Wettkämpfe für Kinder und Schulklassen an, was das Einsatzspektrum erweitert.

Merke Alle vorbestehenden Erkrankungen können während körperlicher Anstrengung aggravieren.

\section{Substanzeinnahme}

Die Einnahme ärztlich verordneter Substanzen muss von eigenständig durch den Läufer angesetzten Substanzen unterschieden werden. Der Übergang von Stoffen auf Grundlage einer medizinischen Indikation zur leistungssteigernden Substanz ist fließend.

\section{Ärztlich verordnete Substanzen}

Die Kombination von sportlicher Aktivität und der Einnahme von Statinpräparaten begünstigt das Auftreten einer Rhabdomyolyse [58]. Östrogene im Rahmen der Kontrazeption oder Osteoporosetherapie erhöhen das Risiko einer Thrombose [51]. Patienten mit bestehender antikoagulatorischer Medikation haben im Rahmen eines Traumas ein erhöhtes Risiko für Blutungskomplikationen.

\section{Eigenständig angesetzte \\ Substanzen}

Küster et al. zeigten, dass $49 \%$ der Marathonläufer vor dem Wettkampf NSAR, Cyclooxygenase(COX)-2-Inhibitoren oder Paracetamol zur Erhöhung der Schmerzschwelle einnehmen [49]. Insbesondere Einsteiger und Amateursportler mit nachlassender Leistungsfähigkeit gehören zur Risikogruppe [49]. Die Inhibition der Prostaglandinsynthese durch NSAR und COX2-Inhibitoren kann zu Magengeschwüren, gastrointestinalen Blutungen, kardiovaskulären Ereignissen sowie Schädigung der Nierenfunktion führen $[49,59]$.

\section{Leistungssteigernde Substanzen}

Verschreibungspflichtige Substanzen ohne medizinische Indikation werden von 7,1\% aller Sportler bei Wettkampfveranstaltungen eingenommen [60]. Fehlende Kontrollen sowie schnelle und einfache Verfügbarkeit über digitale Bezugsquellen führen zu großer Verbreitung von leis- tungssteigernden Substanzen unter professionellen und Amateurathleten [61]. Das Spektrum leistungssteigernder Substanzen und ihrer Nebenwirkungen ist breit und nicht Gegenstand des vorliegenden Artikels. Medizinische Kontakte treten insbesondere im Rahmen der Einnahme von Stimulanzien auf, die über sympathoadrenerge Reaktionen eine zusätzliche Leistungssteigerung bewirken. Überschießende sympathomimetische Effekte begünstigen Tachykardien, Palpitationen, Herzrhythmusstörungen, Rhabdomyolyse und Myokardischämien [61, 62]. Langfristig angewandte anabole androgene Steroide (AAS) steigern das Risiko für Herzinsuffizienz und -infarkt sowie Leberschädigungen. Zudem werden AAS häufig in Kombination mit Insulin eingenommen und können zur Hypoglykämie führen [61].

\section{Äußere Einflussfaktoren}

Laufveranstaltungen gehen häufig mit Rahmenveranstaltungen einher. Die medizinische Versorgung einer hohen Anzahl an Zuschauern muss daher ebenfalls sichergestellt werden. Eine zusätzliche Risikobewertung der Rahmenveranstaltung mit dem von Aarau modifizierten Maurer-Schema stellt die Grundlage für die Bereitstellung ausreichender Ressourcen dar [63]. Durch umfangreiche Beeinträchtigung des Straßenverkehrs im Rahmen von Laufveranstaltungen kommt es zu verlängerten Anfahrtszeiten für Rettungsmittel [64]. Ein erhöhtes Patientenaufkommen verlängert die Wartebzw. Versorgungszeit in Notaufnahmen für die gesamte Bevölkerung [64]. Für das Auftreten eines Massenanfalls von Verletzten (Terrorismus) muss die präklinische und klinische Versorgung einer großen Anzahl an Verletzten kurzfristig sichergestellt werden.

Oberhalb einer Lufttemperatur von $29^{\circ} \mathrm{C}$ nehmen rettungsdienstliche Kontakte, Kollaps und Rennabbrüche zu [65-67]. Viele Autoren verwenden zur Beurteilung von Hitzestressbelastung auf den Menschen die sog. „wet bulb globe temperature" (WBGT), die Effekte von Temperatur, Luftfeuchtigkeit, Windgeschwindigkeit und Sonnenstrahlung auf den Menschen berücksichtigt [68]. Die WBGT kann unkompliziert mittels Hitzestress- messgeräten gemessen werden. Roberts et al. empfehlen vor dem Hintergrund der Häufung medizinischer Zwischenfälle eine Absage der Laufveranstaltung $a b$ einer WBGT von $21^{\circ} \mathrm{C}$ [69].

Merke Die Temperatur sollte während einer Laufveranstaltung mit adäquaten Methoden gemessen und kommuniziert werden. Der Rettungsdienst muss bei hohen Außentemperaturen auf entsprechende Erkrankungsmuster (z.B. Hitzschlag, Kollaps) vorbereitet sein.

\section{COVID-19}

Auf Grundlage einer Computersimulation wird das Übertragungsrisiko für COVID19 bei Laufveranstaltungen als sehr gering eingeschätzt. Starts in Kleingruppen reduzieren das Risiko weiter [70].

\section{Klinik und Diagnose}

Unspezifische Symptome wie Erbrechen, Fieber, Kollaps, Muskelschmerzen, Übelkeit und Schwäche sind die führenden Symptome bei Laufveranstaltungen ( $\mathbf{0}$ Tab. 3). Wegweisend für die Erstversorgung und eine weiterführende klinische Therapie sind eine sorgfältige Anamnese und die vollständige Überprüfung der Vitalfunktionen. Einer „Bagatellisierung" der Symptomatik durch den Läufer selbst sollte entgegengewirkt werden. Häufig kommt es nach dem Zieleinlauf innerhalb eines kurzen Zeitraums zu einem großen Patientenaufkommen, was bei der Planung der sanitätsdienstlichen Kräfte beachtet werden muss.

Merke Kernpunkte der präklinischen Versorgung von Patienten bei Laufveranstaltungen sind die genaue Anamnese sowie die gewissenhafte Überprüfung und Sicherung der Vitalfunktionen. Nach dem Zieleinlauf zeigen sich häufig erst Symptome von Erschöpfung.

\section{Fazit für die Praxis}

- Der Kollaps infolge einer klassischen orthostatischen Dysregulation stellt die häufigste Ursache für medizinische Kontakte bei Langstreckenläufen dar.

- Unspezifische Symptome wie Erbrechen, Fieber, Kollaps, Muskelschmerzen, Übel- 
keit und Schwäche sind die führenden Symptome bei Laufveranstaltungen. Hauptaufgabe des Rettungsdiensts ist die Beurteilung der Schwere eines vorliegenden Krankheitsbilds und die rasche Zuführung in ein geeignetes Krankenhaus.

- Bewusstseinsstörungen und generalisierte Krampfanfälle sind schwerwiegende Komplikationen und können bei hohem Patientenaufkommen leicht übersehen werden.

- Der Kreislaufstillstand ist ein seltenes Ereignis bei Laufveranstaltungen.

- Die Hälfte der Marathonläufer nimmt nichtverschreibungspflichtige Substanzen zur Erhöhung der Schmerzschwelle ein.

- Das Flüssigkeitsmanagement stellt eine besondere Herausforderung unter präklinischen Bedingungen dar.

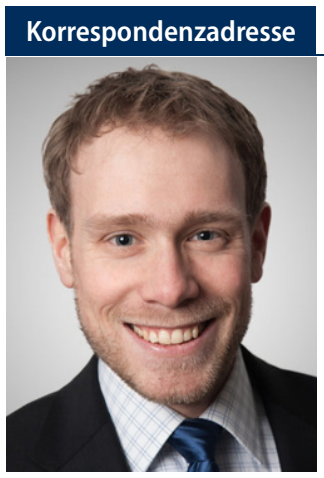

Daniel C. Schroeder, MD

Klinik X - Anästhesie, Intensivmedizin und Notfallmedizin, Bundeswehrzentralkrankenhaus Koblenz

Rübenacher Str. 170, 56072 Koblenz,

Deutschland

daniel.schroeder@uk-koeln.de

Funding. Open Access funding enabled and organized by Projekt DEAL.

\section{Einhaltung ethischer Richtlinien}

Interessenkonflikt. S.-R. Finke, C. Jänig, A. Deschler, J. Hanske, H. Herff, J. Hinkelbein, W. Schmidbauer und D.C. Schroeder geben an, dass kein Interessenkonflikt besteht. B.W. Böttiger ist Schatzmeister und Immediate Past Director Science and Research des European Resuscitation Council (ERC); Vorstandsvorsitzender des Deutschen Rates für Wiederbelebung/German Resuscitation Council (GRC); Mitglied im Präsidium der Deutschen Interdisziplinären Vereinigung für Intensiv- und Notfallmedizin (DIVI); Gründer der Deutschen Stiftung Wiederbelebung, Associated Editor des European Journal of Anaesthesiology (EJA); Mitherausgeber der Zeitschrift Resuscitation; Schriftleiter der Zeitschrift Notfall + Rettungsmedizin, Mitherausgeber der Zeitschrift Brazilian Journal of Anesthesiology. Für Vorträge hat er Honorare der folgenden Firmen erhalten: Forum für medizinische Fortbildung (FomF), Baxalta Deutschland GmbH, ZOLL Medical Deutschland GmbH, C. R. Bard GmbH, GS Elektromedizinische Geräte G. Stemple GmbH, Novartis Pharma GmbH, Philips GmbH Market DACH, Bioscience Valuation BSV $\mathrm{GmbH}$.

Für diesen Beitrag wurden von den Autoren keine Studien an Menschen oder Tieren durchgeführt. Für die aufgeführten Studien gelten die jeweils dort angegebenen ethischen Richtlinien.

Open Access. Dieser Artikel wird unter der Creative Commons Namensnennung 4.0 International Lizenz veröffentlicht, welche die Nutzung, Vervielfältigung, Bearbeitung, Verbreitung und Wiedergabe in jeglichem Medium und Format erlaubt, sofern Sie den/die ursprünglichen Autor(en) und die Quelle ordnungsgemäß nennen, einen Link zur Creative Commons Lizenz beifügen und angeben, ob Änderungen vorgenommen wurden.

Die in diesem Artikel enthaltenen Bilder und sonstiges Drittmaterial unterliegen ebenfalls der genannten Creative Commons Lizenz, sofern sich aus der Abbildungslegende nichts anderes ergibt. Sofern das betreffende Material nicht unter der genannten Creative Commons Lizenz steht und die betreffende Handlung nicht nach gesetzlichen Vorschriften erlaubt ist, ist für die oben aufgeführten Weiterverwendungen des $\mathrm{Ma}$ terials die Einwilligung des jeweiligen Rechteinhabers einzuholen.

Weitere Details zur Lizenz entnehmen Sie bitte der Lizenzinformation auf http://creativecommons.org/ licenses/by/4.0/deed.de.

\section{Literatur}

1. SpasovskiG, VanholderR,AllolioB, AnnaneD, BallS, Bichet $D$ et al (2014) Clinical practice guideline on diagnosis and treatment of hyponatraemia. Eur J Endocrinol 170(3):G1-G47

2. Bennett BL, Hew-Butler T, Hoffman MD, Rogers IR, Rosner MH (2013) Wilderness Medical Society practice guidelines for treatment of exerciseassociated hyponatremia. Wilderness Environ Med 24(3):228-240

3. STATISTA GmbH (2020) Marathon in Deutschland. https://www.statista.com. Zugegriffen: 15.05.2020

4. Preuss H (2012) Laufen in Deutschland - Zahlen, Fakten, Daten 2011. https://www.leichtathletik. de/fileadmin/user_upload/08_Laufen/Volks_ und_Strassenlauf/Zahlen__Daten_Fakten 2012.pdf.Zugegriffen: 20.10 .2021

5. Schwellnus MP, Derman EW, Noakes TD (1997) Aetiology of skeletal muscle "cramps" during exercise: a novel hypothesis. J Sports Sci 15(3):277-285

6. Esser M (2018) Muscle Cramps: Superficial Knowledge Versus Evidence. Sportverletz Sportschaden 32(4):260-263

7. Galloway SD (1999) Dehydration, rehydration, and exercise in the heat: rehydration strategies for athletic competition. Can J Appl Physiol 24(2):188-200

8. Figueroa D, Figueroa F, Calvo R (2016) Patellar tendinopathy: diagnosis and treatment. J Am Acad Orthop Surg 24(12):e184-e192

9. Cook JL, Purdam CR (2009) Is tendon pathology a continuum? A pathology model to explain the clinical presentation of load-induced tendinopathy. Br J Sports Med 43(6):409-416

10. Knobloch K, Yoon U, Vogt PM (2008) Acute and overuse injuries correlated to hours of training in master running athletes. Foot Ankle Int 29(7):671-676

11. Singh D, Angel J, Bentley G, Trevino SG (1997) Fortnightly review. Plantar fasciitis. BMJ 315(7101):172-175

12. Schaps KP, Kessler O, Fetzner U (2008) Chirurgie, Orthopädie, Urologie. Springer, Heidelberg. ISBN 978-3-540-46336-8

13. Meyer R-P, Gächter A (2001) Schulterchirurgie in der Praxis. Springer, Heidelberg. ISBN 978-3-64256427-7

14. Lopes AD et al (2012) What are the main runningrelated musculoskeletal injuries? A Systematic Review. Sports Med 42(10):891-905. https://doi. org/10.1007/BF03262301

15. Zutt R, van der Kooi AJ, Linthorst GE, Wanders RJ, de Visser M (2014) Rhabdomyolysis: review of the literature. Neuromuscul Disord 24(8):651-659

16. Hodgson LE, Walter E, Venn RM, Galloway R, Pitsiladis Y, Sardat Fetal (2017) Acute kidney injury associated with endurance events - is it a cause for concern? A systematic review. BMJ Open Sport ExercMed 3(1):e93

17. Saunier J, Chapurlat R (2018) Stress fracture in athletes. Joint Bone Spine 85(3):307-310

18. MacDonald R (1984) Physiotherapy management of marathon musculo-skeletal casualties. $\mathrm{Br} \mathrm{J}$ Sports Med 18(4):283-285

19. Ibanez B, James S, Agewall S, Antunes MJ, Bucciarelli-Ducci C, Bueno H et al (2018) 2017 ESC guidelines for the management of acute myocardial infarction in patients presenting with ST-segment elevation: the task force for the management of acute myocardial infarction in patients presenting with ST-segment elevation of the European society of cardiology (ESC). Eur Heart J39(2):119-177

20. Surgenor S, Uphold RE (1994) Acute hyponatremia in ultra-endurance athletes. Am J Emerg Med 12(4):441-444

21. Knechtle B, Chlíbková D, Nikolaidis P (2019) Die belastungsassoziierte Hyponatriämie im Ausdauersport. Praxis 108(9):615-632. https://doi. org/10.1024/1661-8157/a003261

22. Almond CS, Shin AY, Fortescue EB, Mannix RC, Wypij D, Binstadt BA et al (2005) Hyponatremia among runners in the Boston Marathon. N Engl J Med 352(15):1550-1556

23. Ziegenfuß T (2017) Notfallmedizin, 7. Aufl. Springer, Berlin Heidelberg

24. Travers AH, Perkins GD, Berg RA, Castren M, Considine J, Escalante R et al (2015) Part 3: adult basic life support and automated external defibrillation: 2015 international consensus on cardiopulmonary resuscitation and emergency cardiovascular care science with treatment recommendations. Circulation 132(16 Suppl 1):S51-S83

25. Soar J, Böttiger BW, Carli P, Couper K, Deakin CD, Djärv Tet al (2021) European Resuscitation Council Guidelines 2021: adult advanced life support. Resuscitation 161:115-151

26. Hach-Wunderle V, Konstantinides S, Riess $H$, Wildberger JE, Gerlach H, Noppeney THet al (2015) S2k-Leitlinie zur Diagnostik und Therapie der Venenthrombose und der Lungenembolie Deutschen Gesellschaft für Angiologie. Gesellschaft für Gefäßmedizin e.V. 
27. Nelson PB, Robinson AG, KapoorW, RinaldoJ(1988) Hyponatremia in a Marathoner. Phys Sportsmed 16(10):78-87

28. Braune S, Lücking CH (1997) Orthostatische Hypotonie: Pathophysiologie, Differentialdiagnose und Therapie. Dtsch Arztebl Int 94(50):A-3413

29. Torlincasi AM, Lopez RA, Waseem M (2020) Acute compartment syndrome. StatPearls Publishing, Treasure Island

30. Luning $\mathrm{H}$, Mangelus $\mathrm{C}$, Carlstrom E, Nilson F, Borjesson M (2019) Incidence and characteristics of severe exercise-associated collapse at the world's largest half-marathon. PLoS ONE 14(6):e217465

31. Lier H, Bernhard M, Hossfeld B (2018) Hypovolemic and hemorrhagic shock. Anaesthesist 67(3):225-244

32. de Bruijn J, Winkes $M$, van Eerten $P$, Scheltinga M (2019) Chronisches belastungsbedingtes Kompartmentsyndrom als Ursache anterolateraler Beinschmerzen. Unfallchirurg 122(11):840-847

33. Ghio FE, Pieri M, Agracheva A, Melisurgo G, Ponti A, Serini C (2012) Sudden cardiac arrest in a marathon runner. A case report. HSR Proc Intensive Care Cardiovasc Anesth 4(2):130-132

34. Kim JH, Malhotra R, Chiampas G, d'Hemecourt P, Troyanos C, Cianca J et al (2012) Cardiac arrest during long-distance running races. $\mathrm{N}$ Engl I Med 366(2):130-140

35. Corrado D, Basso C, Thiene G (2012) Sudden cardiac death in athletes: what is the role of screening? Curr Opin Cardiol 27(1):41-48

36. Webner D, DuPrey KM, Drezner JA, Cronholm P, Roberts WO (2012) Sudden cardiac arrest and death in United States marathons. Med Sci Sports Exerc 44(10):1843-1845

37. Maron BJ, Doerer JJ, Haas TS, Tierney DM, Mueller FO (2009) Sudden deaths in young competitive athletes: analysis of 1866 deaths in the United States, 1980-2006. Circulation 119(8):1085-1092

38. Borjesson M, Pelliccia A (2009) Incidence and aetiology of sudden cardiac death in young athletes: an international perspective. $\mathrm{Br} J$ Sports Med 43(9):644-648

39. Siegel AJ (2015) Fatal water intoxication and cardiac arrest in runners during marathons: prevention and treatment based on validated clinical paradigms. Am J Med 128(10):1070-1075

40. Paana T, Jaakkola S, Bamberg K, Saraste A, Tuunainen E, Wittfooth S et al (2019) Cardiac troponin elevations in marathon runners. Role of coronary atherosclerosis and skeletal muscle injury. The MaraCat Study. Int J Cardiol 295:25-28

41. Lott C, Truhláŕ A, Alfonzo A, Barelli A, GonzálezSalvado V, Hinkelbein J et al (2021) European Resuscitation Council Guidelines 2021: cardiac arrest in special circumstances. Resuscitation 161:152-219

42. Till D, Matthias S (2020) Hyponatriämie in der Notaufnahme - eine ernst zu nehmende Elektrolytstörung. Notaufnahme Up2date 2:1-92

43. Davis DP, Videen JS, Marino A, Vilke GM, Dunford JV, Van Camp SP et al (2001) Exercise-associated hyponatremia in marathon runners: a two-year experience.J Jmerg Med 21(1):47-57

44. Pomroy S, Lovell G, Hughes D, Vlahovich N (2020) Intravenous fluids and their use in sport: a position statement from the Australian institute of sport. JSci Med Sport 23(4):322-328

45. Zouhal H, Groussard C, Minter G, Vincent S, Cretual A, Gratas-Delamarche A et al (2011) Inverse relationship between percentage body weight change and finishing time in 643 forty-two-

\section{Medical emergencies during running events}

Background: The number of short- and long-distance running events in Germany is increasing. Running as a popular sport is practiced by a large number of people of different ages, risk groups, and degrees of professionalism, which results in a wide range of medical emergencies.

Objective: The present article elucidates incidence, pathophysiology and therapy of relevant emergencies during running events. Aim was the optimization of work processes of emergency personnel.

Materials and methods: A literature search was conducted in PubMed.

Results: Exercise-associated muscle cramps, gastrointestinal symptoms, collapse, compartment syndrome, and tendinopathy are common clinical manifestations. Cardiac arrest and sudden cardiac death are rare events. Consciousness and seizures are major complications. Disseminated intravascular coagulation, exercise-associated hyponatremia, heat stroke, rhabdomyolysis, and thromboembolism are associated with high morbidity and mortality. Substances increasing pain resilience as well as performance-enhancing substances are popular among amateur and professional runners and are associated with a high incidence of side effects.

Conclusion: General symptoms including vomiting, fever, collapse, muscle-pain, nausea and weakness are the leading symptoms during running events. A careful anamnesis is important for targeted clinical therapy. Symptom control is the main task. Fluid management the most challenging task for healthcare providers in the prehospital setting.

\section{Keywords}

Marathon · Jogging · Sudden cardiac death · Athletic injuries · Cardiopulmonary resuscitation

kilometre marathon runners. Br J Sports Med 45(14):1101-1105

46. Liu SY, Wang Q, Lou YP, Gao Y, Ning B, Song Q et al (2020) Interpretations and comments for expert consensus on the diagnosis and treatment of heat stroke in China. Mil Med Res 7(1):37

47. Tanen D (2019) Hitzekrankheit im Überblick. https://www.msdmanuals.com/de-de/profi/ verletzungen,-vergiftungen/hitzekrankheit/ hitzekrankheit-im-\%C3\%BCberblick. Zugegriffen: 05.03.2021

48. Gilchrist T, Haileyesus T, Murphy M, Collins C, Yard E (2010) Heat illness among high school athletes-United States, 2005-2009. Centers for Disease Control and Prevention

49. Kuster M, Renner B, Oppel P, Niederweis U, Brune K (2013) Consumption of analgesics before a marathon and the incidence of cardiovascular, gastrointestinal and renal problems: a cohort study. BMJ Open 3(4):e002090. https://doi.org/10. 1136/bmjopen-2012-002090

50. Feldkamp T, Witzke $O$ (2021) Acute kidney injury. Nephrologe 16(2):65

51. Hull CM, Harris JA (2013) Cardiology patient page. Venous thromboembolism and marathon athletes. Circulation 128(25):e469-e471

52. Cauley K, Wright P (2005) Iliac vein compression and pulmonary embolism in a long distance runner: computed tomography and magnetic resonance imaging - a case report. Angiology 56(1):87-91

53. Chan KL, Davies RA, Chambers RJ (1984) Coronary thrombosis and subsequent lysis after a marathon J Am Coll Cardiol 4(6):1322-1325

54. Gaudard A, Varlet-Marie E, Monnier JF, Janbon C Quere I, Bressolle F et al (2002) Exercise-induced central retinal vein thrombosis: possible involve- ment of hemorheological disturbances. A case report. Clin Hemorheol Microcirc 27(2):115-122

55. Herold J, Mitrasch A, Lorenz E, Lodes U, Tanev I, Braun-Dullaeus $R$ et al (2018) Vomiting and collapse of a 28-year-old male long distance runner in middle European Summer. Internist 59(8):850-856

56. de Oliveira EP, Burini RC, Jeukendrup A (2014) Gastrointestinal complaints during exercise: prevalence, etiology, and nutritional recommendations. Sports Med 44(Suppl 1):S79-S85

57. Peters HP, Wiersma JW, Koerselman J, Akkermans LM, Bol E, Mosterd WL et al (2000) The effect of a sports drink on gastroesophageal reflux during a run-bike-run test. Int J Sports Med 21(1):65-70

58. Collins R, Reith C, Emberson J, Armitage J, Baigent C, Blackwell L et al (2016) Interpretation of the evidence for the efficacy and safety of statin therapy. Lancet 388(10059):2532-2561

59. Alaranta A, Alaranta H, Helenius I (2008) Use of prescription drugs in athletes. Sports Med 38(6):449-463

60. Robert-Koch-Institut (2011) KOLIBRI - Studie zum Konsum leistungsbeeinflussender Mittel in Alltag und Freizeit

61. Siegmund-Schultze N (2013) Leistungsbeeinflussende Substanzen im Breiten- und Freizeitsport: Trainieren mit allen Mitteln. Dtsch Arztebl Int 110(29-30):A-1422 / B-1248/C-1230

62. Huckins DS, Lemons MF (2013) Myocardial ischemia associated with clenbuterol abuse: report of two cases. J Emerg Med 44(2):444-449

63. Hanno P, Klaus M (2005) Gefahrenabwehr bei Großveranstaltungen. Stumpf \& Kossendey

64. Jena $A B$, Mann NC, Wedlund LN, Olenski A (2017) Delays in emergency care and mortality during major U.S. marathons. N Engl J Med 376(15):1441-1450 


\section{In eigener Sache}

65. Khorram-Manesh A, Löf T, Börjesson M, Nilson F, Thorsson S, Lindberg F et al (2019) Profiling collapsing half marathon runners-emerging risk factors: results from Gothenburg half marathon. Sports 8(1):2. https://doi.org/10.3390/sports8010002

66. Carlström E, Borjesson M, Palm G, KhorramManesh A, Lindberg F, Holmer B et al (2019) Medical emergencies during a half marathon race - the influence of weather. Int J Sports Med 40(5):312-316

67. Roberts WO (2007) Heat and cold: what does the environment do to marathon injury? Sports Med 37(4-5):400-403

68. Werner A (2018) Beurteilung von Hitzebelastungen mit dem Klimasummenmaß Wet Bulb Globe Temperature - Praktische Anwendung im militärischen Alltag. Wehrmedizinische Monatsschrift 62(10):367

69. Roberts WO (2010) Determining a "do not start" temperature for a marathon on the basis of adverse outcomes. Med Sci Sports Exerc 42(2):226-232

70. Übertragung von COVID-19 bei Laufveranstaltungen sehr selten - Meldungen - Marathon. www. marathon4you.de.Zugegriffen: 20.10.2021

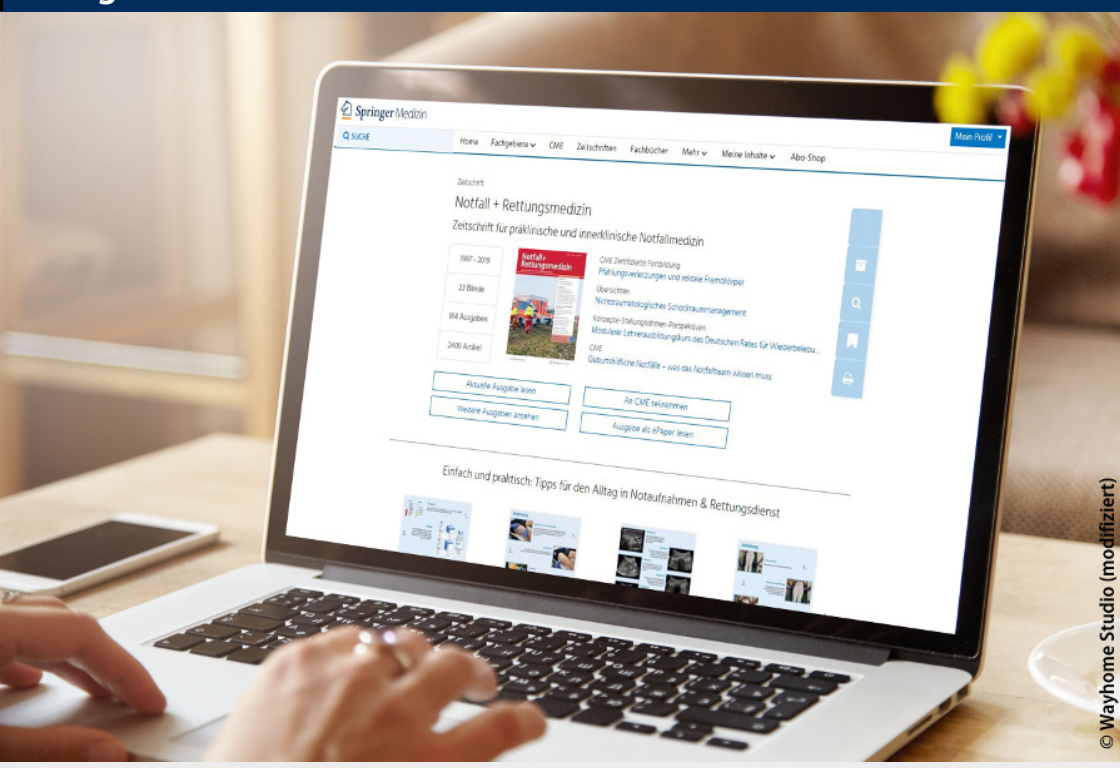

\section{Auch online Zugang zu allen Beiträgen von Notfall+Rettungsmedizin}

Wussten Sie, dass Sie als Abonnent*in dieser Zeitschrift automatisch online Zugriffsrechte auf das gesamte Beitragsarchiv haben?

Durch Ihr Abonnement der Notfall+Rettungsmedizin erhalten Sie acht mal im Jahr die aktuelle Ausgabe der Zeitschrift nach Hause geliefert.

Doch damit nicht genug: Sie haben mit dem Abonnement außerdem Zugriff auf das gesamte Online-Archiv Ihrer Zeitschrift.

Und so einfach geht es:

Registrieren Sie sich einmal über www.springermedizin.de/register:

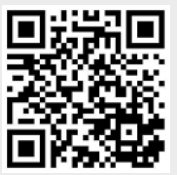

Über diesen QR-Code schnel und einfach registrieren

Bei der Registrierung geben Sie einfach Ihren Vor- und Nachname und Lieferadresse wie beim Abonnement der Zeitschrift (siehe Adressaufkleber auf Ihrem Heft) an. So kann im System die Zugehörigkeit zu Ihrer Zeitschrift sichergestellt werden.

Sollten Fragen oder Probleme auftauchen, wenden Sie sich einfach an den Kundenservice:

kundenservice@springermedizin.de
Aufgrund des Heilmittelwerbegesetzes dürfen die Inhalte der Website nur medizinischen Fachkreisen zur Verfügung gestellt werden. Bei der Anmeldung bitten wir Sie deshalb einen Berufsnachweis vorzulegen.

Bei Mediziner*innen mit Mitgliedschaft in der deutschen Ärztekammer reicht die einheitliche Fortbildungsnummer (EFN). Als Angehörige ${ }^{*} r$ des nichtärztlichen Rettungsdienstfachpersonals oder Pflegepersonal schicken Sie eine Bestätigung der Arbeitsstelle. Sind Sie Student*in, dann bitte den Studiennachweis mit Angabe des Studiengangs ganz unkompliziert an kundenservice@springermedizin.de.

Mit Benutzername und Passwort haben Sie außerdem Zugang zu den freien Inhalten auf den Seiten von: https://www.springermedizin.de/ https://www.aerztezeitung.de/ 